\title{
Generalizations of Hu-type inequalities and their applications
}

\author{
Jingfeng Tian ${ }^{\mathrm{a}, *}$, Zhen-Hang Yang ${ }^{\mathrm{b}}$ \\ ${ }^{a}$ College of Science and Technology, North China Electric Power University, Baoding, Hebei Province, 071051, P. R. China. \\ ${ }^{b}$ Customer Service Center, State Grid Zhejiang Electric Power Research Institute, Hangzhou, Zhejiang Province, 310009, P. R. \\ China.
}

Communicated by C. Zaharia

\begin{abstract}
In this paper, we present some new generalizations of Hu-type inequalities, and then we obtain some new generalizations and refinements of Hölder's inequality. (C)2017 All rights reserved.
\end{abstract}

Keywords: Hölder's inequality, Hu-type inequality, generalization, refinement. 2010 MSC: 26D15, 26D10.

\section{Introduction}

The classical Hölder's inequality states that if $a_{k} \geqslant 0, b_{k} \geqslant 0(k=1,2, \cdots, n), p>0, q>0$ and $\frac{1}{p}+\frac{1}{q}=1$, then

$$
\sum_{k=1}^{n} a_{k} b_{k} \leqslant\left(\sum_{k=1}^{n} a_{k}^{p}\right)^{\frac{1}{p}}\left(\sum_{k=1}^{n} b_{k}^{q}\right)^{\frac{1}{q}} .
$$

The inequality (1.1) is reversed for $p<1(p \neq 0)$; (For $p<1$, we assume that $\left.a_{k}, b_{k}>0\right)$.

Hölder's inequality plays a very important role in both theory and applications. This classical inequality has been widely studied by many authors, and it has motivated a large number of research papers involving different proofs, various generalizations, variations and applications (see e.g., [1, 6-14, 16-18] and the references therein).

Among various refinements of (1.1), Hu in [4] established the following interesting theorems.

Theorem 1.1. Let $p \geqslant q>0, \frac{1}{p}+\frac{1}{q}=1$, let $A_{k}, B_{k} \geqslant 0(k=1,2, \cdots, n)$, and let $1-e_{r}+e_{s} \geqslant 0$ $(r, s=1,2, \cdots, n)$. Then

$$
\begin{aligned}
\sum_{k=1}^{n} A_{k} B_{k} \leqslant & \left(\sum_{k=1}^{n} B_{k}^{q}\right)^{\frac{1}{q}-\frac{1}{p}}\left\{\left[\left(\sum_{k=1}^{n} B_{k}^{q}\right)\left(\sum_{k=1}^{n} A_{k}^{p}\right)\right]^{2}\right. \\
& \left.-\left[\left(\sum_{k=1}^{n} B_{k}^{q} e_{k}\right)\left(\sum_{k=1}^{n} A_{k}^{p}\right)-\left(\sum_{k=1}^{n} B_{k}^{q}\right)\left(\sum_{k=1}^{n} A_{k}^{p} e_{k}\right)\right]^{2}\right\}^{\frac{1}{2 p}} .
\end{aligned}
$$

\footnotetext{
*Corresponding author

Email addresses: tianjf@ncepu.edu.cn (Jingfeng Tian), yzhkm@163.com (Zhen-Hang Yang)
} 
The integral form is as follows:

Theorem 1.2. Let $f(x), g(x), e(x)$ be integrable functions defined on $[a, b]$ and $f(x), g(x) \geqslant 0,1-e(x)+e(y) \geqslant 0$ for all $x, y \in[a, b]$, and let $p \geqslant q>0, \frac{1}{p}+\frac{1}{q}=1$. Then

$$
\begin{aligned}
\int_{a}^{b} f(x) g(x) d x \leqslant & \left(\int_{a}^{b} g^{q}(x) d x\right)^{\frac{1}{q}-\frac{1}{p}}\left[\left(\int_{a}^{b} f^{p}(x) d x \int_{a}^{b} g^{q}(x) d x\right)^{2}\right. \\
& \left.-\left(\int_{a}^{b} f^{p}(x) e(x) d x \int_{a}^{b} g^{q}(x) d x-\int_{a}^{b} f^{p}(x) d x \int_{a}^{b} g^{q}(x) e(x) d x\right)^{2}\right]^{\frac{1}{2 p}} .
\end{aligned}
$$

Later, Tian in [7] gave the reversed versions of Hu's inequalities (1.2) and (1.3).

Theorem 1.3. Let $p<0, q>0, \frac{1}{p}+\frac{1}{q}=1$, let $A_{k}>0, B_{k} \geqslant 0(k=1,2, \cdots, n)$, and let $1-e_{r}+e_{s} \geqslant 0$ $(\mathrm{r}, \mathrm{s}=1,2, \cdots, \mathrm{n})$. Then

$$
\begin{aligned}
\sum_{k=1}^{n} A_{k} B_{k} \geqslant & \left(\sum_{k=1}^{n} B_{k}^{q}\right)^{\frac{1}{q}-\frac{1}{p}}\left\{\left[\left(\sum_{k=1}^{n} B_{k}^{q}\right)\left(\sum_{k=1}^{n} A_{k}^{p}\right)\right]^{2}\right. \\
& \left.-\left[\left(\sum_{k=1}^{n} B_{k}^{q} e_{k}\right)\left(\sum_{k=1}^{n} A_{k}^{p}\right)-\left(\sum_{k=1}^{n} B_{k}^{q}\right)\left(\sum_{k=1}^{n} A_{k}^{p} e_{k}\right)\right]^{2}\right\}^{\frac{1}{2 p}} .
\end{aligned}
$$

The integral form is as follows:

Theorem 1.4. Let $\mathrm{f}(\mathrm{x}), \mathrm{g}(\mathrm{x}), \mathrm{e}(\mathrm{x})$ be integrable functions defined on $[\mathrm{a}, \mathrm{b}]$ and $\mathrm{f}(\mathrm{x})>0, \mathrm{~g}(\mathrm{x}) \geqslant 0,1-\mathrm{e}(\mathrm{x})+$ $e(y) \geqslant 0$ for all $x, y \in[a, b]$, and let $p<0, q>0, \frac{1}{p}+\frac{1}{q}=1$. Then

$$
\begin{aligned}
\int_{a}^{b} f(x) g(x) d x \geqslant & \left(\int_{a}^{b} g^{q}(x) d x\right)^{\frac{1}{q}-\frac{1}{p}}\left[\left(\int_{a}^{b} f^{p}(x) d x \int_{a}^{b} g^{q}(x) d x\right)^{2}\right. \\
& \left.-\left(\int_{a}^{b} f^{p}(x) e(x) d x \int_{a}^{b} g^{q}(x) d x-\int_{a}^{b} f^{p}(x) d x \int_{a}^{b} g^{q}(x) e(x) d x\right)^{2}\right]^{\frac{1}{2 p}} .
\end{aligned}
$$

In 2007, Wu [16] presented the generalizations of Hu's results, as follows:

Theorem 1.5. Let $A_{r} \geqslant 0, B_{r}>0(r=1,2, \cdots, n)$, let $1-e_{r}+e_{s} \geqslant 0(r, s=1,2, \cdots, n)$, and let $p \geqslant q>$ $0, \mu=\min \left\{\frac{1}{p}+\frac{1}{q}, 1\right\}$. Then

$$
\begin{aligned}
\sum_{r=1}^{n} A_{r} B_{r} \leqslant & n^{1-\mu}\left(\sum_{r=1}^{n} B_{r}^{q}\right)^{\frac{1}{q}-\frac{1}{p}}\left\{\left[\left(\sum_{r=1}^{n} B_{r}^{q}\right)\left(\sum_{r=1}^{n} A_{r}^{p}\right)\right]^{2}\right. \\
& \left.-\left[\left(\sum_{r=1}^{n} B_{r}^{q} e_{r}\right)\left(\sum_{r=1}^{n} A_{r}^{p}\right)-\left(\sum_{r=1}^{n} B_{r}^{q}\right)\left(\sum_{r=1}^{n} A_{r}^{p} e_{r}\right)\right]^{2}\right\}^{\frac{1}{2 p}} .
\end{aligned}
$$

Theorem 1.6. Let $f(x), g(x), e(x)$ be integrable functions defined on $[a, b]$ and $f(x) \geqslant 0, g(x)>0,1-e(x)+$ $e(y) \geqslant 0$ for all $x, y \in[a, b]$, and let $p \geqslant q>0, \frac{1}{p}+\frac{1}{q} \leqslant 1$. Then

$$
\begin{aligned}
\int_{a}^{b} f(x) g(x) d x \leqslant & (b-a)^{1-\frac{1}{p}-\frac{1}{q}}\left(\int_{a}^{b} g^{q}(x) d x\right)^{\frac{1}{q}-\frac{1}{p}}\left[\left(\int_{a}^{b} g^{q}(x) d x \int_{a}^{b} f^{p}(x) d x\right)^{2}\right. \\
& \left.-\left(\int_{a}^{b} g^{q}(x) e(x) d x \int_{a}^{b} f^{p}(x) d x-\int_{a}^{b} g^{q}(x) d x \int_{a}^{b} f^{p}(x) e(x) d x\right)^{2}\right]^{\frac{1}{2 p}} .
\end{aligned}
$$

In 2012, Tian [8] proved the following reversed versions of inequalities (1.6) and (1.7).

Theorem 1.7. Let $A_{r}>0, B_{r}>0(r=1,2, \cdots, n)$, let $1-e_{r}+e_{s} \geqslant 0(r, s=1,2, \cdots, n)$, and let $q<$ 
$0, \frac{1}{p}+\frac{1}{q} \geqslant 0, \mu=\max \left\{\frac{1}{p}+\frac{1}{q}, 1\right\}, \lambda=\max \left\{\frac{1}{q},-1\right\}$. Then

$$
\sum_{r=1}^{n} A_{r} B_{r} \leqslant n^{1-\mu}\left(\sum_{r=1}^{n} A_{r}^{p}\right)^{\frac{1}{p}}\left(\sum_{r=1}^{n} B_{r}^{q}\right)^{\frac{1}{q}}\left[1-\left(\frac{\sum_{r=1}^{n} A_{r} B_{r} e_{r}}{\sum_{r=1}^{n} A_{r} B_{r}}-\frac{\sum_{r=1}^{n} B_{r}^{q} e_{r}}{\sum_{r=1}^{n} B_{r}^{q}}\right)^{2}\right]^{\frac{\lambda}{2}} .
$$

Theorem 1.8. Let $f(x), g(x), e(x)$ be integrable functions defined on $[a, b]$ and $f(x)>0, g(x)>0,1-e(x)+$ $e(y) \geqslant 0$ for all $x, y \in[a, b]$, and let $q<0, \frac{1}{p}+\frac{1}{q} \geqslant 1, \lambda=\max \left\{-1, \frac{1}{q}\right\}$. Then

$$
\begin{aligned}
\int_{a}^{b} f(x) g(x) d x \geqslant & (b-a)^{1-\frac{1}{p}-\frac{1}{q}}\left(\int_{a}^{b} f^{p}(x) d x\right)^{\frac{1}{p}}\left(\int_{a}^{b} g^{q}(x) d x\right)^{\frac{1}{q}} \\
& \times\left[1-\left(\frac{\int_{a}^{b} f(x) g(x) e(x) d x}{\int_{a}^{b} f(x) g(x) d x}-\frac{\int_{a}^{b} g^{q}(x) e(x) d x}{\int_{a}^{b} g^{q}(x) d x}\right)^{2}\right]^{\frac{\lambda}{2}} .
\end{aligned}
$$

Later, in 2013, Tian and $\mathrm{Hu}$ [13] presented another reversed versions of inequalities (1.6) and (1.7).

Theorem 1.9. Let $A_{r} \geqslant 0, B_{r}>0(r=1,2, \cdots, n)$, let $1-e_{r}+e_{s} \geqslant 0(r, s=1,2, \cdots, n)$, and let $q<0, p>$ $0, \rho=\max \left\{\frac{1}{p}+\frac{1}{q}, 1\right\}$. Then

$$
\begin{aligned}
\sum_{r=1}^{n} A_{r} B_{r} \geqslant & n^{1-\rho}\left(\sum_{r=1}^{n} A_{r}^{p}\right)^{\frac{1}{p}-\frac{1}{q}}\left\{\left[\left(\sum_{r=1}^{n} A_{r}^{p}\right)\left(\sum_{r=1}^{n} B_{r}^{q}\right)\right]^{2}\right. \\
& \left.-\left[\left(\sum_{r=1}^{n} A_{r}^{p} e_{r}\right)\left(\sum_{r=1}^{n} B_{r}^{q}\right)-\left(\sum_{r=1}^{n} A_{r}^{p}\right)\left(\sum_{r=1}^{n} B_{r}^{q} e_{r}\right)\right]^{2}\right\}^{\frac{1}{2 q}} .
\end{aligned}
$$

Theorem 1.10. Let $f(x), g(x), e(x)$ be integrable functions defined on $[a, b]$ and $f(x), g(x)>0,1-e(x)+e(y) \geqslant$ 0 for all $x, y \in[a, b]$, and let $\mathrm{q}<0, \frac{1}{\mathrm{p}}+\frac{1}{\mathrm{q}} \geqslant 1$. Then

$$
\begin{aligned}
\int_{a}^{b} f(x) g(x) d x \geqslant & (b-a)^{1-\frac{1}{p}-\frac{1}{q}}\left(\int_{a}^{b} f^{p}(x) d x\right)^{\frac{1}{p}-\frac{1}{q}}\left[\left(\int_{a}^{b} f^{p}(x) d x \int_{a}^{b} g^{q}(x) d x\right)^{2}\right. \\
& \left.-\left(\int_{a}^{b} f^{p}(x) e(x) d x \int_{a}^{b} g^{q}(x) d x-\int_{a}^{b} f^{p}(x) d x \int_{a}^{b} g^{q}(x) e(x) d x\right)^{2}\right]^{\frac{1}{2 q}} .
\end{aligned}
$$

In 2011, Tian in [7] gave the following generalizations of inequalities (1.2) and (1.3).

Theorem 1.11. Let $A_{n j} \geqslant 0, \sum_{n} A_{n j}^{\lambda_{j}}<\infty(j=1,2, \cdots, k), \lambda_{1} \geqslant \lambda_{2} \geqslant \cdots \geqslant \lambda_{k}>0, \sum_{j=1}^{k} \frac{1}{\lambda_{j}}=1$, and let $1-e_{n}+e_{m} \geqslant 0, \sum_{n}\left|e_{n}\right|<\infty$. If $k$ is even number, then

$$
\begin{aligned}
\sum_{n} \prod_{j=1}^{k} A_{n j} \leqslant & \prod_{j=1}^{\frac{k}{2}}\left\{( \sum _ { n } A _ { n ( 2 j - 1 ) } ^ { \lambda _ { 2 j - 1 } } ) ^ { \frac { 2 } { \lambda _ { 2 j - 1 } } - \frac { 2 } { \lambda _ { 2 j } } } \left[\left(\left(\sum_{n} A_{n(2 j-1)}^{\lambda_{2 j-1}}\right)\left(\sum_{n} A_{n(2 j)}^{\lambda_{2 j}}\right)\right)^{2}\right.\right. \\
& \left.\left.-\left(\left(\sum_{n} A_{n(2 j-1)}^{\lambda_{2 j-1}} e_{n}\right)\left(\sum_{n} A_{n(2 j)}^{\lambda_{2 j}}\right)-\sum_{n}\left(A_{n(2 j-1)}^{\lambda_{2 j-1}}\right)\left(\sum_{n} A_{n(2 j)}^{\lambda_{2 j}} e_{n}\right)\right)^{2}\right]^{\frac{1}{\lambda_{2 j}}}\right\} .
\end{aligned}
$$

If $\mathrm{k}$ is odd number, then

$$
\begin{aligned}
\sum_{n} \prod_{j=1}^{k} A_{n j} \leqslant & \left(\sum_{n} A_{n k}^{\lambda_{k}}\right)^{\frac{2}{\lambda_{k}}} \prod_{j=1}^{\frac{k-1}{2}}\left\{\left(\sum_{n} A_{n(2 j-1)}^{\lambda_{2 j-1}}\right)^{\frac{2}{\lambda_{2 j-1}}-\frac{2}{\lambda_{2 j}}}\right. \\
\times & {\left[\left(\left(\sum_{n} A_{n(2 j-1)}^{\lambda_{2 j-1}}\right)\left(\sum_{n} A_{n(2 j)}^{\lambda_{2 j}}\right)\right)^{2}-\left(\left(\sum_{n} A_{n(2 j-1)}^{\lambda_{2 j-1}} e_{n}\right)\left(\sum_{n} A_{n(2 j)}^{\lambda_{2 j}}\right)\right.\right.} \\
& \left.\left.\left.-\sum_{n}\left(A_{n(2 j-1)}^{\lambda_{2 j-1}}\right)\left(\sum_{n} A_{n(2 j)}^{\lambda_{2 j}} e_{n}\right)\right)^{2}\right]^{\frac{1}{\lambda_{2 j}}}\right\} .
\end{aligned}
$$


Theorem 1.12. Let $A_{k 1} \geqslant 0, A_{k j}>0,(j=1,2, \cdots, m, k=1,2, \cdots, n), \sum_{j=1}^{m} \frac{1}{\lambda_{j}}=1$, and let $1-e_{r}+e_{s} \geqslant$ $0(r, s=1,2, \cdots, n)$. If $\lambda_{1}>0, \lambda_{j}<0,(j=2,3, \cdots, m)$, then

$$
\begin{aligned}
\sum_{k=1}^{n} \prod_{j=1}^{m} A_{k j} \geqslant & \left(\sum_{k=1}^{n} A_{k 1}^{\lambda_{1}}\right)^{\frac{1}{\lambda_{1}}-\sum_{j=2}^{m} \frac{1}{\lambda_{j}}} \prod_{j=2}^{m}\left\{\left[\left(\sum_{k=1}^{n} A_{k 1}^{\lambda_{1}}\right)\left(\sum_{k=1}^{n} A_{k j}^{\lambda_{j}}\right)\right]^{2}\right. \\
& \left.-\left[\left(\sum_{k=1}^{n} A_{k 1}^{\lambda_{1}} e_{k}\right)\left(\sum_{k=1}^{n} A_{k j}^{\lambda_{j}}\right)-\left(\sum_{k=1}^{n} A_{k 1}^{\lambda_{1}}\right)\left(\sum_{k=1}^{n} A_{k j}^{\lambda_{j}} e_{k}\right)\right]^{2}\right\}^{\frac{1}{2 \lambda_{j}}} .
\end{aligned}
$$

The classic Hölder inequality is an important cornerstone in different branches of modern mathematics such as classical real and complex analysis, probability and statistics, numerical analysis, qualitative theory of differential equations. It is also a bridge to help solve problems into depth. The Hu's inequality (1.2), which was put forward by $\mathrm{Hu}$ in [4], improves the Hölder inequality exquisitely. The mathematical reviews [5] calls it "an extraordinary, outstanding and new inequality". The classic Hölder inequality is playing a basic role in mathematics and can be applied in a wide range of areas, while the function of the Hu's inequality is the same.

The purpose of this work is to give some new generalizations of the above Hu-type inequalities (1.2), (1.3), (1.4), (1.5), (1.6), (1.7) and (1.8), (1.9), (1.10), (1.11), (1.12). Moreover, the obtained results will be applied to improve Hölder's inequality and Popoviciu-type inequality which is due to Wu and Debnath.

\section{Main results}

We begin this section with some lemmas, which will be used in the sequel.

Lemma 2.1 ([2]). If $x>-1, \alpha>1$ or $\alpha<0$, then

$$
(1+x)^{\alpha} \geqslant 1+\alpha x
$$

The inequality is reversed for $0<\alpha<1$.

Lemma 2.2 ([3]). If $x_{i} \geqslant 0, \lambda_{i}>0, i=1,2, \cdots, n, 0<p \leqslant 1$, then

$$
\sum_{i=1}^{n} \lambda_{i} x_{i}^{p} \leqslant\left(\sum_{i=1}^{n} \lambda_{i}\right)^{1-p}\left(\sum_{i=1}^{n} \lambda_{i} x_{i}\right)^{p} .
$$

The inequality is reversed for $\mathrm{p} \geqslant 1$ or $\mathrm{p}<0$.

Lemma 2.3 (Generalized Hölder's inequality [15]).

(a) Let $A_{i j} \geqslant 0(i=1,2, \cdots, n, j=1,2, \cdots, m)$, and let $\lambda_{j}>0$ with $\sum_{j=1}^{m} \frac{1}{\lambda_{j}} \geqslant 1$. Then

$$
\sum_{i=1}^{n} \prod_{j=1}^{m} A_{i j} \leqslant \prod_{j=1}^{m}\left(\sum_{i=1}^{n} A_{i j}\right)^{\frac{1}{\lambda_{j}}} .
$$

(b) Let $A_{i j}>0(i=1,2, \cdots, n, j=1,2, \cdots, m)$, and let $\lambda_{1}>0, \lambda_{j}<0(j=2,3, \cdots, m)$ with $\sum_{j=1}^{m} \frac{1}{\lambda_{j}} \leqslant 1$. Then

$$
\sum_{i=1}^{n} \prod_{j=1}^{m} A_{i j} \geqslant \prod_{j=1}^{m}\left(\sum_{i=1}^{n} A_{i j}\right)^{\frac{1}{\lambda_{j}}} .
$$

(c) Let $A_{i j}>0(i=1,2, \cdots, n, j=1,2, \cdots, m)$, and let $\lambda_{j}<0(j=1,2, \cdots, m)$. Then

$$
\sum_{i=1}^{n} \prod_{j=1}^{m} A_{i j} \geqslant \prod_{j=1}^{m}\left(\sum_{i=1}^{n} A_{i j}\right)^{\frac{1}{\lambda_{j}}}
$$


Next, we generalize the inequalities (1.2), (1.3), (1.6), (1.7), (1.10) and (1.11) as follows.

Theorem 2.4. Let $A_{r j} \geqslant 0,(r=1,2, \cdots, n, j=1,2, \cdots, k), \lambda_{1} \geqslant \lambda_{2} \geqslant \cdots \geqslant \lambda_{k}>0$, let $\rho=\min \left\{\sum_{j=1}^{k} \frac{1}{\lambda_{j}}, 1\right\}$, and let $1-e_{r}+e_{s} \geqslant 0,(s=1,2, \cdots, n)$. If $k$ is even, then

$$
\begin{aligned}
\sum_{r=1}^{n} \prod_{j=1}^{k} A_{r j} \leqslant & n^{1-\rho} \prod_{j=1}^{\frac{k}{2}}\left\{( \sum _ { r = 1 } ^ { n } A _ { r ( 2 j - 1 ) } ^ { \lambda _ { 2 j - 1 } } ) ^ { \frac { 1 } { \lambda _ { 2 j - 1 } } - \frac { 1 } { \lambda _ { 2 j } } } \left[\left(\left(\sum_{r=1}^{n} A_{r(2 j-1)}^{\lambda_{2 j-1}}\right)\left(\sum_{r=1}^{n} A_{r(2 j)}^{\lambda_{2 j}}\right)\right)^{2}\right.\right. \\
& \left.\left.-\left(\left(\sum_{r=1}^{n} A_{r(2 j-1)}^{\lambda_{2 j-1}} e_{r}\right)\left(\sum_{r=1}^{n} A_{r(2 j)}^{\lambda_{2 j}}\right)-\left(\sum_{r=1}^{n} A_{r(2 j-1)}^{\lambda_{2 j-1}}\right)\left(\sum_{r=1}^{n} A_{r(2 j)}^{\lambda_{2 j}} e_{r}\right)\right)^{2}\right]^{\frac{1}{2 \lambda_{2 j}}}\right\} .
\end{aligned}
$$

If $\mathrm{k}$ is odd, then

$$
\begin{aligned}
\sum_{r=1}^{n} \prod_{j=1}^{k} A_{r j} \leqslant & n^{1-\rho}\left(\sum_{r=1}^{n} A_{r k}^{\lambda_{k}}\right)^{\frac{1}{\lambda_{k}}} \prod_{j=1}^{\frac{k-1}{2}}\left\{\left(\sum_{r=1}^{n} A_{r(2 j-1)}^{\lambda_{2 j-1}}\right)^{\frac{1}{\lambda_{2 j-1}}-\frac{1}{\lambda_{2 j}}}\right. \\
& \times\left[\left(\left(\sum_{r=1}^{n} A_{r(2 j-1)}^{\lambda_{2 j-1}}\right)\left(\sum_{r=1}^{n} A_{r(2 j)}^{\lambda_{2 j}}\right)\right)^{2}-\left(\left(\sum_{r=1}^{n} A_{r(2 j-1)}^{\lambda_{2 j-1}} e_{r}\right)\left(\sum_{r=1}^{n} A_{r(2 j)}^{\lambda_{2 j}}\right)\right.\right. \\
& \left.\left.\left.-\left(\sum_{r=1}^{n} A_{r(2 j-1)}^{\lambda_{2 j-1}}\right)\left(\sum_{r=1}^{n} A_{r(2 j)}^{\lambda_{2 j}} e_{r}\right)\right)^{2}\right]^{\frac{1}{2 \lambda_{2 j}}}\right\} .
\end{aligned}
$$

Proof. Preforming some simple computations, we have

$$
\begin{aligned}
\sum_{r=1}^{n}\left(\prod_{j=1}^{k} A_{r j}\right) & \sum_{s=1}^{n}\left(\prod_{i=1}^{k} A_{s i}\right)\left(1-e_{r}+e_{s}\right) \\
= & \sum_{r=1}^{n} \sum_{s=1}^{n}\left(\prod_{j=1}^{k} A_{r j}\right)\left(\prod_{i=1}^{k} A_{s i}\right)-\sum_{r=1}^{n} \sum_{s=1}^{n}\left(\prod_{j=1}^{k} A_{r j}\right)\left(\prod_{i=1}^{k} A_{s i}\right) e_{r} \\
& +\sum_{r=1}^{n} \sum_{s=1}^{n}\left(\prod_{j=1}^{k} A_{r j}\right)\left(\prod_{i=1}^{k} A_{s i}\right) e_{s} \\
= & \left(\sum_{r=1}^{n} \prod_{j=1}^{k} A_{r j}\right)^{2} .
\end{aligned}
$$

Case 1. Let $\lambda_{1}>\lambda_{2}>\cdots>\lambda_{k}>0$.

Subcase (1): When $k$ is even, and $\sum_{j=1}^{k} \frac{1}{\lambda_{j}} \geqslant 1$. From Lemma 2.2, we have

$$
\begin{aligned}
\sum_{r=1}^{n} & \left(\prod_{j=1}^{k} A_{r j}\right) \sum_{s=1}^{n}\left(\prod_{i=1}^{k} A_{s i}\right)\left(1-e_{r}+e_{s}\right)^{\sum_{j=1}^{k} \frac{1}{\lambda_{j}}} \\
& =\sum_{r=1}^{n} \sum_{s=1}^{n}\left(\prod_{j=1}^{k} A_{r j}\right)\left(\prod_{i=1}^{k} A_{s i}\right)\left(1-e_{r}+e_{s}\right)^{\sum_{j=1}^{k} \frac{1}{\lambda_{j}}} \\
& \geqslant\left[\sum_{r=1}^{n} \sum_{s=1}^{n}\left(\prod_{j=1}^{k} A_{r j}\right)\left(\prod_{i=1}^{k} A_{s i}\right)\right]^{1-\sum_{j=1}^{k} \frac{1}{\lambda_{j}}}\left[\sum_{r=1}^{n} \sum_{s=1}^{n}\left(\prod_{j=1}^{k} A_{r j}\right)\left(\prod_{i=1}^{k} A_{s i}\right)\left(1-e_{r}+e_{s}\right)\right]^{\sum_{j=1}^{k} \frac{1}{\lambda_{j}}}(2 . \\
& =\left[\sum_{r=1}^{n} \sum_{s=1}^{n}\left(\prod_{j=1}^{k} A_{r j}\right)\left(\prod_{i=1}^{k} A_{s i}\right)\right]^{1-\sum_{j=1}^{k} \frac{1}{\lambda_{j}}}\left[\sum_{r=1}^{n} \sum_{s=1}^{n}\left(\prod_{j=1}^{k} A_{r j}\right)\left(\prod_{i=1}^{k} A_{s i}\right)\right]^{\sum_{j=1}^{k} \frac{1}{\lambda_{j}}}
\end{aligned}
$$




$$
\begin{aligned}
& =\sum_{r=1}^{n} \sum_{s=1}^{n}\left(\prod_{j=1}^{k} A_{r j}\right)\left(\prod_{i=1}^{k} A_{s i}\right) \\
& =\left[\sum_{r=1}^{n}\left(\prod_{j=1}^{k} A_{r j}\right)\right]^{2} .
\end{aligned}
$$

Moreover, in view of $\sum_{j=1}^{k} \frac{1}{\lambda_{j}} \geqslant 1$, from inequality (2.2), we deduce

$$
\begin{aligned}
& \sum_{r=1}^{n}\left(\prod_{j=1}^{k} A_{r j}\right) \sum_{s=1}^{n}\left(\prod_{i=1}^{k} A_{s i}\right)\left(1-e_{r}+e_{s}\right)^{\sum_{j=1}^{k} \frac{1}{\lambda_{j}}} \\
& =\sum_{r=1}^{n}\left(\prod_{j=1}^{k} A_{r j}\right) \sum_{s=1}^{n} \prod_{i=1}^{k} A_{s i}\left(1-e_{r}+e_{s}\right)^{\frac{1}{\lambda_{i}}} \\
& \leqslant \sum_{r=1}^{n}\left(\prod_{j=1}^{k} A_{r j}\right)\left[\prod_{i=1}^{k}\left(\sum_{s=1}^{n} A_{s i}^{\lambda_{i}}\left(1-e_{r}+e_{s}\right)\right)^{\frac{1}{\lambda_{i}}}\right] \\
& =\sum_{r=1}^{n}\left\{\prod _ { j = 1 } ^ { \frac { k } { 2 } } \left[\left(A_{r(2 j-1)}^{\lambda_{2 j-1}} \sum_{s=1}^{n} A_{s(2 j-1)}^{\lambda_{2 j-1}}\left(1-e_{r}+e_{s}\right)\right)^{\frac{1}{\lambda_{2 j-1}}-\frac{1}{\lambda_{2 j}}}\left(A_{r(2 j-1)}^{\lambda_{2 j-1}} \sum_{s=1}^{n} A_{s(2 j)}^{\lambda_{2 j}}\left(1-e_{r}+e_{s}\right)\right)^{\frac{1}{\lambda_{2 j}}}\right.\right. \\
& \left.\left.\times\left(A_{r(2 j)}^{\lambda_{2 j}} \sum_{s=1}^{n} A_{s(2 j-1)}^{\lambda_{(2 j-1)}}\left(1-e_{r}+e_{s}\right)\right)^{\frac{1}{\lambda_{2 j}}}\right]\right\} .
\end{aligned}
$$

Hence, according to $\left(\frac{1}{\lambda_{1}}-\frac{1}{\lambda_{2}}\right)+\frac{1}{\lambda_{2}}+\frac{1}{\lambda_{2}}+\left(\frac{1}{\lambda_{3}}-\frac{1}{\lambda_{4}}\right)+\frac{1}{\lambda_{4}}+\frac{1}{\lambda_{4}}+\cdots+\left(\frac{1}{\lambda_{k-1}}-\frac{1}{\lambda_{k}}\right)+\frac{1}{\lambda_{k}}+\frac{1}{\lambda_{k}} \geqslant 1$, applying inequality (2.2) on the right side of (2.8), we have

$$
\begin{aligned}
& \sum_{r=1}^{n}\left(\prod_{j=1}^{k} A_{r j}\right) \sum_{s=1}^{n}\left(\prod_{i=1}^{k} A_{s i}\right)\left(1-e_{r}+e_{s}\right)^{\sum_{j=1}^{k} \frac{1}{\lambda_{j}}} \\
& \leqslant \prod_{j=1}^{\frac{k}{2}}\left[\left(\sum_{r=1}^{n} A_{r(2 j-1)}^{\lambda_{2 j-1}} \sum_{s=1}^{n} A_{s(2 j-1)}^{\lambda_{2 j-1}}\left(1-e_{r}+e_{s}\right)\right)^{\frac{1}{\lambda_{2 j-1}}-\frac{1}{\lambda_{2 j}}}\right. \\
& \left.\times\left(\sum_{r=1}^{n} A_{r(2 j-1)}^{\lambda_{2 j-1}} \sum_{s=1}^{n} A_{s(2 j)}^{\lambda_{2 j}}\left(1-e_{r}+e_{s}\right)\right)^{\frac{1}{\lambda_{2 j}}}\left(\sum_{r=1}^{n} A_{r(2 j)}^{\lambda_{2 j}} \sum_{s=1}^{n} A_{s(2 j-1)}^{\lambda_{2 j-1}}\left(1-e_{r}+e_{s}\right)\right)^{\frac{1}{\lambda_{2 j}}}\right] \\
& =\prod_{j=1}^{\frac{k}{2}}\left\{( \sum _ { r = 1 } ^ { n } A _ { r ( 2 j - 1 ) } ^ { \lambda _ { 2 j - 1 } } ) ^ { \frac { 2 } { \lambda _ { 2 j - 1 } } - \frac { 2 } { \lambda _ { 2 j } } } \left[\left(\sum_{r=1}^{n} \sum_{s=1}^{n} A_{r(2 j-1)}^{\lambda_{2 j-1}} A_{s(2 j)}^{\lambda_{2 j}}\left(1-e_{r}+e_{s}\right)\right)\right.\right. \\
& \left.\left.\times\left(\sum_{r=1}^{n} \sum_{s=1}^{n} A_{r(2 j)}^{\lambda_{2 j}} A_{s(2 j-1)}^{\lambda_{2 j-1}}\left(1-e_{r}+e_{s}\right)\right)\right]^{\frac{1}{\lambda_{2 j}}}\right\} \\
& =\prod_{j=1}^{\frac{k}{2}}\left\{( \sum _ { r = 1 } ^ { n } A _ { r ( 2 j - 1 ) } ^ { \lambda _ { 2 j - 1 } } ) ^ { \frac { 2 } { \lambda _ { 2 j - 1 } } - \frac { 2 } { \lambda _ { 2 j } } } \left[\left(\sum_{r=1}^{n} A_{r(2 j-1)}^{\lambda_{2 j-1}} \sum_{s=1}^{n} A_{s(2 j)}^{\lambda_{2 j}}\right.\right.\right. \\
& \left.-\sum_{r=1}^{n} A_{r(2 j-1)}^{\lambda_{2 j-1}} e_{r} \sum_{s=1}^{n} A_{s(2 j)}^{\lambda_{2 j}}+\sum_{r=1}^{n} A_{r(2 j-1)}^{\lambda_{2 j-1}} \sum_{s=1}^{n} A_{s(2 j)}^{\lambda_{2 j}} e_{s}\right) \\
& \left.\left.\times\left(\sum_{r=1}^{n} A_{r(2 j)}^{\lambda_{2 j}} \sum_{s=1}^{n} A_{s(2 j-1)}^{\lambda_{2 j-1}}-\sum_{r=1}^{n} A_{r(2 j)}^{\lambda_{2 j}} e_{r} \sum_{s=1}^{n} A_{s(2 j-1)}^{\lambda_{2 j-1}}+\sum_{r=1}^{n} A_{r(2 j)}^{\lambda_{2 j}} \sum_{s=1}^{n} A_{s(2 j-1)}^{\lambda_{2 j-1}} e_{s}\right)\right]^{\frac{1}{\lambda_{2 j}}}\right\}
\end{aligned}
$$




$$
\begin{aligned}
= & \prod_{j=1}^{\frac{k}{2}}\left\{( \sum _ { r = 1 } ^ { n } A _ { r ( 2 j - 1 ) } ^ { \lambda _ { 2 j - 1 } } ) ^ { \frac { 2 } { \lambda _ { 2 j - 1 } } - \frac { 2 } { \lambda _ { 2 j } } } \left[\left(\left(\sum_{r=1}^{n} A_{r(2 j-1)}^{\lambda_{2 j-1}}\right)\left(\sum_{r=1}^{n} A_{r(2 j)}^{\lambda_{2 j}}\right)\right)^{2}\right.\right. \\
& \left.\left.-\left(\left(\sum_{r=1}^{n} A_{r(2 j-1)}^{\lambda_{2 j-1}} e_{r}\right)\left(\sum_{r=1}^{n} A_{r(2 j)}^{\lambda_{2 j}}\right)-\left(\sum_{r=1}^{n} A_{r(2 j-1)}^{\lambda_{2 j-1}}\right)\left(\sum_{r=1}^{n} A_{r(2 j)}^{\lambda_{2 j}} e_{r}\right)\right)^{2}\right]^{\frac{1}{\lambda_{2 j}}}\right\} .
\end{aligned}
$$

Combining inequalities (2.7) and (2.9) yields inequality (2.4).

Subcase (2): When $k$ is even, and $\sum_{j=1}^{k} \frac{1}{\lambda_{j}}<1$. Let $\sum_{j=1}^{k} \frac{1}{\lambda_{j}}=\beta(0<\beta<1)$, which implies that $\sum_{j=1}^{k} \frac{1}{\beta \lambda_{j}}=1$. From inequality (2.2) we find

$$
\begin{aligned}
\sum_{r=1}^{n}( & \left.\prod_{j=1}^{k} A_{r j}\right) \sum_{s=1}^{n}\left(\prod_{i=1}^{k} A_{s i}\right)\left(1-e_{r}+e_{s}\right) \\
= & \sum_{r=1}^{n}\left(\prod_{j=1}^{k} A_{r j}\right) \sum_{s=1}^{n} \prod_{i=1}^{k} A_{s i}\left(1-e_{r}+e_{s}\right)^{\frac{1}{\beta \lambda_{i}}} \\
\leqslant & \sum_{r=1}^{n}\left(\prod_{j=1}^{k} A_{r j}\right)\left[\prod_{i=1}^{k}\left(\sum_{s=1}^{n} A_{s i}^{\beta \lambda_{i}}\left(1-e_{r}+e_{s}\right)\right)^{\frac{1}{\beta \lambda_{i}}}\right] \\
= & \sum_{r=1}^{n}\left\{\prod _ { j = 1 } ^ { \frac { k } { 2 } } \left[\left(A_{r(2 j-1)}^{\beta \lambda_{2 j-1}} \sum_{s=1}^{n} A_{s(2 j-1)}^{\beta \lambda_{2 j-1}}\left(1-e_{r}+e_{s}\right)\right)^{\frac{1}{\beta \lambda_{2 j-1}}-\frac{1}{\beta \lambda_{2 j}}}\right.\right. \\
& \left.\left.\times\left(A_{r(2 j-1)}^{\beta \lambda_{2 j-1}} \sum_{s=1}^{n} A_{s(2 j)}^{\beta \lambda_{2 j}}\left(1-e_{r}+e_{s}\right)\right)^{\frac{1}{\beta \lambda_{2 j}}}\left(A_{r(2 j)}^{\beta \lambda_{2 j}} \sum_{s=1}^{n} A_{s(2 j-1)}^{\beta \lambda_{2 j-1}}\left(1-e_{r}+e_{s}\right)\right)^{\frac{1}{\beta \lambda_{2 j}}}\right]\right\} .
\end{aligned}
$$

Consequently, in view of $\left(\frac{1}{\beta \lambda_{1}}-\frac{1}{\beta \lambda_{2}}\right)+\frac{1}{\beta \lambda_{2}}+\frac{1}{\beta \lambda_{2}}+\left(\frac{1}{\beta \lambda_{3}}-\frac{1}{\beta \lambda_{4}}\right)+\frac{1}{\beta \lambda_{4}}+\frac{1}{\beta \lambda_{4}}+\cdots+\left(\frac{1}{\beta \lambda_{k-1}}-\frac{1}{\beta \lambda_{k}}\right)+\frac{1}{\beta \lambda_{k}}+$ $\frac{1}{\beta \lambda_{k}}=1$, and applying inequality (2.2) on the right side of (2.10), we have

$$
\begin{aligned}
& \sum_{r=1}^{n}\left(\prod_{j=1}^{k} A_{r j}\right) \sum_{s=1}^{n}\left(\prod_{i=1}^{k} A_{s i}\right)\left(1-e_{r}+e_{s}\right) \\
& \leqslant \prod_{j=1}^{\frac{k}{2}}\left[\left(\sum_{r=1}^{n} A_{r(2 j-1)}^{\beta \lambda_{2 j-1}} \sum_{s=1}^{n} A_{s(2 j-1)}^{\beta \lambda_{2 j-1}}\left(1-e_{r}+e_{s}\right)\right)^{\frac{1}{\beta \lambda_{2 j-1}}-\frac{1}{\beta \lambda_{2 j}}}\right. \\
& \left.\times\left(\sum_{r=1}^{n} A_{r(2 j-1)}^{\beta \lambda_{2 j-1}} \sum_{s=1}^{n} A_{s(2 j)}^{\beta \lambda_{2 j}}\left(1-e_{r}+e_{s}\right)\right)^{\frac{1}{\beta \lambda_{2 j}}}\left(\sum_{r=1}^{n} A_{r(2 j)}^{\beta \lambda_{2 j}} \sum_{s=1}^{n} A_{s(2 j-1)}^{\beta \lambda_{2 j-1}}\left(1-e_{r}+e_{s}\right)\right)^{\frac{1}{\beta \lambda_{2 j}}}\right] \\
& =\prod_{j=1}^{\frac{k}{2}}\left[\left(\sum_{r=1}^{n} \sum_{s=1}^{n} A_{r(2 j-1)}^{\beta \lambda_{2 j-1}} A_{s(2 j-1)}^{\beta \lambda_{2 j-1}}\left(1-e_{r}+e_{s}\right)\right)^{\frac{1}{\beta \lambda_{2 j-1}}-\frac{1}{\beta \lambda_{2 j}}}\right. \\
& \left.\times\left(\sum_{r=1}^{n} \sum_{s=1}^{n} A_{r(2 j-1)}^{\beta \lambda_{2 j-1}} A_{s(2 j)}^{\beta \lambda_{2 j}}\left(1-e_{r}+e_{s}\right)\right)^{\frac{1}{\beta \lambda_{2 j}}}\left(\sum_{r=1}^{n} \sum_{s=1}^{n} A_{r(2 j)}^{\beta \lambda_{2 j}} A_{s(2 j-1)}^{\beta \lambda_{2 j-1}}\left(1-e_{r}+e_{s}\right)\right)^{\frac{1}{\beta \lambda_{2 j}}}\right] .
\end{aligned}
$$

Additionally, applying Lemma 2.2 on the right side of (2.11), we find

$$
\prod_{j=1}^{\frac{k}{2}}\left[\left(\sum_{r=1}^{n} \sum_{s=1}^{n} A_{r(2 j-1)}^{\beta \lambda_{2 j-1}} A_{s(2 j-1)}^{\beta \lambda_{2 j-1}}\left(1-e_{r}+e_{s}\right)\right)^{\frac{1}{\beta \lambda_{2 j-1}}-\frac{1}{\beta \lambda_{2 j}}}\right.
$$




$$
\begin{aligned}
& \left.\times\left(\sum_{r=1}^{n} \sum_{s=1}^{n} A_{r(2 j-1)}^{\beta \lambda_{2 j-1}} A_{s(2 j)}^{\beta \lambda_{2 j}}\left(1-e_{r}+e_{s}\right)\right)^{\frac{1}{\beta \lambda_{2 j}}}\left(\sum_{r=1}^{n} \sum_{s=1}^{n} A_{r(2 j)}^{\beta \lambda_{2 j}} A_{s(2 j-1)}^{\beta \lambda_{2 j-1}}\left(1-e_{r}+e_{s}\right)\right)^{\frac{1}{\beta \lambda_{2 j}}}\right] \\
& \leqslant \prod_{j=1}^{\frac{k}{2}}\left[\left(\sum_{r=1}^{n} \sum_{s=1}^{n}\left(1-e_{r}+e_{s}\right)\right)^{(1-\beta)\left(\frac{1}{\beta \lambda_{2 j}-1}-\frac{1}{\beta \lambda_{2 j}}\right)}\right. \\
& \times\left(\sum_{r=1}^{n} \sum_{s=1}^{n} A_{r(2 j-1)}^{\lambda_{2 j-1}} A_{s(2 j-1)}^{\lambda_{2 j-1}}\left(1-e_{r}+e_{s}\right)\right)^{\frac{1}{\lambda_{2 j-1}}-\frac{1}{\lambda_{2 j}}} \\
& \left.\times\left(\sum_{r=1}^{n} \sum_{s=1}^{n}\left(1-e_{r}+e_{s}\right)\right)^{\frac{1-\beta}{\beta \lambda_{2 j}}}\left(\sum_{r=1}^{n} \sum_{s=1}^{n} A_{r(2 j-1)}^{\lambda_{2 j-1}} A_{s(2 j)}^{\lambda_{2 j}}\left(1-e_{r}+e_{s}\right)\right)^{\frac{1}{\lambda_{2 j}}}\right) \\
& \left.\times\left(\sum_{r=1}^{n} \sum_{s=1}^{n}\left(1-e_{r}+e_{s}\right)\right)^{\frac{1-\beta}{\beta \lambda_{2 j}}}\left(\sum_{r=1}^{n} \sum_{s=1}^{n} A_{r(2 j)}^{\lambda_{2 j}} A_{s(2 j-1)}^{\lambda_{2 j-1}}\left(1-e_{r}+e_{s}\right)\right)^{\frac{1}{\lambda_{2 j}}}\right] \\
& =\left(\sum_{r=1}^{n} \sum_{s=1}^{n}\left(1-e_{r}+e_{s}\right)\right)^{1-\beta} \prod_{j=1}^{\frac{k}{2}}\left[\left(\sum_{r=1}^{n} \sum_{s=1}^{n} A_{r(2 j-1)}^{\lambda_{2 j-1}} A_{s(2 j-1)}^{\lambda_{2 j-1}}\left(1-e_{r}+e_{s}\right)\right)^{\frac{1}{\lambda_{2 j-1}}-\frac{1}{\lambda_{2 j}}}\right. \\
& \left.\times\left(\sum_{r=1}^{n} \sum_{s=1}^{n} A_{r(2 j-1)}^{\lambda_{2 j-1}} A_{s(2 j)}^{\lambda_{2 j}}\left(1-e_{r}+e_{s}\right)\right)^{\frac{1}{\lambda_{2 j}}}\left(\sum_{r=1}^{n} \sum_{s=1}^{n} A_{r(2 j)}^{\lambda_{2 j}} A_{s(2 j-1)}^{\lambda_{2 j-1}}\left(1-e_{r}+e_{s}\right)\right)^{\frac{1}{\lambda_{2 j}}}\right] \\
& =n^{2-2 \beta} \prod_{j=1}^{\frac{k}{2}}\left\{\left(\sum_{r=1}^{n} A_{r(2 j-1)}^{\lambda_{2 j-1}}\right)^{\frac{2}{\lambda_{2 j-1}-\frac{2}{\lambda_{2 j}}}}\right. \\
& \left.\times\left[\left(\sum_{r=1}^{n} \sum_{s=1}^{n} A_{r(2 j-1)}^{\lambda_{2 j-1}} A_{s(2 j)}^{\lambda_{2 j}}\left(1-e_{r}+e_{s}\right)\right)\left(\sum_{r=1}^{n} \sum_{s=1}^{n} A_{r(2 j)}^{\lambda_{2 j}} A_{s(2 j-1)}^{\lambda_{2 j-1}}\left(1-e_{r}+e_{s}\right)\right)\right]^{\frac{1}{\lambda_{2 j}}}\right\} \\
& =n^{2-2 \beta} \prod_{j=1}^{\frac{k}{2}}\left\{( \sum _ { r = 1 } ^ { n } A _ { r ( 2 j - 1 ) } ^ { \lambda _ { 2 j - 1 } } ) ^ { \frac { 2 } { \lambda _ { 2 j - 1 } } - \frac { 2 } { \lambda _ { 2 j } } } \left[\left(\sum_{r=1}^{n} A_{r(2 j-1)}^{\lambda_{2 j-1}} \sum_{s=1}^{n} A_{s(2 j)}^{\lambda_{2 j}}\right.\right.\right. \\
& \left.-\sum_{r=1}^{n} A_{r(2 j-1)}^{\lambda_{2 j-1}} e_{r} \sum_{s=1}^{n} A_{s(2 j)}^{\lambda_{2 j}}+\sum_{r=1}^{n} A_{r(2 j-1)}^{\lambda_{2 j-1}} \sum_{s=1}^{n} A_{s(2 j)}^{\lambda_{2 j}} e_{s}\right) \\
& \left.\left.\times\left(\sum_{r=1}^{n} A_{r(2 j)}^{\lambda_{2 j}} \sum_{s=1}^{n} A_{s(2 j-1)}^{\lambda_{2 j-1}}-\sum_{r=1}^{n} A_{r(2 j)}^{\lambda_{2 j}} e_{r} \sum_{s=1}^{n} A_{s(2 j-1)}^{\lambda_{2 j-1}}+\sum_{r=1}^{n} A_{r(2 j)}^{\lambda_{2 j}} \sum_{s=1}^{n} A_{s(2 j-1)}^{\lambda_{2 j-1}} e_{s}\right)\right]^{\frac{1}{\lambda_{2 j}}}\right\} \\
& =n^{2-2 \beta} \prod_{j=1}^{\frac{k}{2}}\left\{( \sum _ { r = 1 } ^ { n } A _ { r ( 2 j - 1 ) } ^ { \lambda _ { 2 j - 1 } } ) ^ { \frac { 2 } { \lambda _ { 2 j - 1 } } - \frac { 2 } { \lambda _ { 2 j } } } \left[\left(\left(\sum_{r=1}^{n} A_{r(2 j-1)}^{\lambda_{2 j-1}}\right)\left(\sum_{r=1}^{n} A_{r(2 j)}^{\lambda_{2 j}}\right)\right)^{2}\right.\right. \\
& \left.\left.-\left(\left(\sum_{r=1}^{n} A_{r(2 j-1)}^{\lambda_{2 j-1}} e_{r}\right)\left(\sum_{r=1}^{n} A_{r(2 j)}^{\lambda_{2 j}}\right)-\left(\sum_{r=1}^{n} A_{r(2 j-1)}^{\lambda_{2 j-1}}\right)\left(\sum_{r=1}^{n} A_{r(2 j)}^{\lambda_{2 j}} e_{r}\right)\right)^{2}\right]^{\frac{1}{\lambda_{2 j}}}\right\} \text {. }
\end{aligned}
$$

Combining inequalities (2.6), (2.11), and (2.12) leads to inequality (2.4) immediately.

Subcase (3): When $k$ is odd, and $\sum_{j=1}^{k} \frac{1}{\lambda_{j}} \geqslant 1$. By the same method as in the above Subcase (1), we have the inequality (2.5).

Subcase (4): When $k$ is odd, and $\sum_{j=1}^{k} \frac{1}{\lambda_{j}}<1$. By the same method as in the above Subcase (2), we have the inequality (2.5).

Case 2. When $\lambda_{1} \geqslant \lambda_{2} \geqslant \cdots \geqslant \lambda_{k}>0$, at least one of the equalities holds. By the same way as in Case 1, we can obtain the desired results. The proof of Theorem 2.4 is completed. 
From Theorem 2.4 and Lemma 2.1, we obtain the generalizations and refinements of the generalized Hölder's inequality (2.2) as follows.

Corollary 2.5. Let $A_{r j}, \lambda_{j}, e_{r}$ be as in Theorem 2.4 , let $\rho=\min \left\{\sum_{j=1}^{k} \frac{1}{\lambda_{j}}, 1\right\}$, and let $\sum_{r=1}^{n} A_{r j}^{\lambda_{j}} \neq 0$. If $k$ is even, then

$$
\sum_{r=1}^{n} \prod_{j=1}^{k} A_{r j} \leqslant n^{1-\rho}\left[\prod_{j=1}^{k}\left(\sum_{r=1}^{n} A_{r j}^{\lambda_{j}}\right)^{\frac{1}{\lambda_{j}}}\right]\left\{\prod_{j=1}^{\frac{k}{2}}\left[1-\frac{1}{2 \lambda_{2 j}}\left(\frac{\sum_{r=1}^{n} A_{r(2 j-1)}^{\lambda_{2 j-1}} e_{r}}{\sum_{r=1}^{n} A_{r(2 j-1)}^{\lambda_{2 j-1}}}-\frac{\sum_{r=1}^{n} A_{r(2 j)}^{\lambda_{2 j}} e_{r}}{\sum_{r=1}^{n} A_{r(2 j)}^{\lambda_{2 j}}}\right)^{2}\right]\right\} .
$$

If $\mathrm{k}$ is odd, then

$$
\sum_{r=1}^{n} \prod_{j=1}^{k} A_{r j} \leqslant n^{1-\rho}\left[\prod_{j=1}^{k}\left(\sum_{r=1}^{n} A_{r j}^{\lambda_{j}}\right)^{\frac{1}{\lambda_{j}}}\right]\left\{\prod_{j=1}^{\frac{k-1}{2}}\left[1-\frac{1}{2 \lambda_{2 j}}\left(\frac{\sum_{r=1}^{n} A_{r(2 j-1)}^{\lambda_{2 j-1}} e_{r}}{\sum_{r=1}^{n} A_{r(2 j-1)}^{\lambda_{2 j-1}}}-\frac{\sum_{r=1}^{n} A_{r(2 j)}^{\lambda_{2 j}} e_{r}}{\sum_{r=1}^{n} A_{r(2 j)}^{\lambda_{2 j}}}\right)^{2}\right]\right\} .
$$

Proof. We only need to prove the inequality (2.13). The proof of inequality (2.14) is similar. From inequality (2.4), we obtain

$$
\sum_{r=1}^{n} \prod_{j=1}^{k} A_{r j} \leqslant n^{1-\rho}\left[\prod_{j=1}^{k}\left(\sum_{r=1}^{n} A_{r j}^{\lambda_{j}}\right)^{\frac{1}{\lambda_{j}}}\right]\left\{\prod_{j=1}^{\frac{k}{2}}\left[1-\left(\frac{\sum_{r=1}^{n} A_{r(2 j-1)}^{\lambda_{2 j-1}} e_{r}}{\sum_{r=1}^{n} A_{r(2 j-1)}^{\lambda_{2 j-1}}}-\frac{\sum_{r=1}^{n} A_{r(2 j)}^{\lambda_{2 j}} e_{r}}{\sum_{r=1}^{n} A_{r(2 j)}^{\lambda_{2 j}}}\right)^{2}\right]^{\frac{1}{2 \lambda_{2 j}}}\right\} .
$$

Furthermore, preforming some simple computations, we have

$$
\left|\frac{\sum_{r=1}^{n} A_{r(2 j-1)}^{\lambda_{2 j-1}} e_{r}}{\sum_{r=1}^{n} A_{r(2 j-1)}^{\lambda_{2 j-1}}}-\frac{\sum_{r=1}^{n} A_{r(2 j)}^{\lambda_{2 j}} e_{r}}{\sum_{r=1}^{n} A_{r(2 j)}^{\lambda_{2 j}}}\right|<1
$$

Consequently, from Lemma 2.1 and the inequalities (2.15) and (2.16), we have the desired inequality (2.13). The proof of Corollary 2.5 is complete.

Theorem 2.6. Let $\lambda_{1} \geqslant \lambda_{2} \geqslant \cdots \geqslant \lambda_{k}>0, \sum_{j=1}^{k} \frac{1}{\lambda_{j}} \leqslant 1$, let $F_{j}(x)$, e(x) be nonnegative integrable functions defined on $[a, b]$, and let $1-e(x)+e(y) \geqslant 0$. If $k$ is even, then

$$
\begin{aligned}
\int_{a}^{b} \prod_{j=1}^{k} F_{j}(x) d x \leqslant & (b-a)^{1-\sum_{j=1}^{k} \frac{1}{\lambda_{j}}} \prod_{j=1}^{\frac{k}{2}}\left\{( \int _ { a } ^ { b } F _ { 2 j - 1 } ^ { \lambda _ { 2 j - 1 } } ( x ) d x ) ^ { \frac { 1 } { \lambda _ { 2 j - 1 } } - \frac { 1 } { \lambda _ { 2 j } } } \left[\left(\int_{a}^{b} F_{2 j-1}^{\lambda_{2 j-1}}(x) d x \int_{a}^{b} F_{2 j}^{\lambda_{2 j}}(x) d x\right)^{2}\right.\right. \\
& \left.\left.-\left(\int_{a}^{b} F_{2 j-1}^{\lambda_{2 j-1}}(x) e(x) d x \int_{a}^{b} F_{2 j}^{\lambda_{2 j}}(x) d x-\int_{a}^{b} F_{2 j-1}^{\lambda_{2 j-1}}(x) d x \int_{a}^{b} F_{2 j}^{\lambda_{2 j}}(x) e(x) d x\right)^{2}\right]^{\frac{1}{2 \lambda_{2 j}}}\right\} .
\end{aligned}
$$

If $\mathrm{k}$ is odd, then

$$
\begin{aligned}
\int_{a}^{b} \prod_{j=1}^{k} F_{j}(x) d x \leqslant & (b-a)^{1-\sum_{j=1}^{k} \frac{1}{\lambda_{j}}}\left(\int_{a}^{b} F_{k}^{\lambda_{k}}(x) d x\right)^{\frac{1}{\lambda_{k}}} \\
& \times \prod_{j=1}^{\frac{k-1}{2}}\left\{( \int _ { a } ^ { b } F _ { 2 j - 1 } ^ { \lambda _ { 2 j - 1 } } ( x ) d x ) ^ { \frac { 1 } { \lambda _ { 2 j - 1 } } - \frac { 1 } { \lambda _ { 2 j } } } \left[\left(\int_{a}^{b} F_{2 j-1}^{\lambda_{2 j-1}}(x) d x \int_{a}^{b} F_{2 j}^{\lambda_{2 j}}(x) d x\right)^{2}\right.\right. \\
& \left.\left.-\left(\int_{a}^{b} F_{2 j-1}^{\lambda_{2 j-1}}(x) e(x) d x \int_{a}^{b} F_{2 j}^{\lambda_{2 j}}(x) d x-\int_{a}^{b} F_{2 j-1}^{\lambda_{2 j-1}}(x) d x \int_{a}^{b} F_{2 j}^{\lambda_{2 j}}(x) e(x) d x\right)^{2}\right]^{\frac{1}{2 \lambda_{2 j}}}\right\} .
\end{aligned}
$$


Proof. For any positive integer $n$, we choose an equidistant partition of $[a, b]$ as

$$
\begin{gathered}
a<a+\frac{b-a}{n}<\cdots<a+\frac{b-a}{n} r<\cdots<a+\frac{b-a}{n}(n-1)<b, \\
x_{r}=a+\frac{b-a}{n} r, \quad \Delta x_{r}=\frac{b-a}{n}, \quad r=1,2, \cdots, n .
\end{gathered}
$$

Case (a). When $k$ is even, by the inequality (2.4) we have

$$
\begin{aligned}
\sum_{r=1}^{n} \prod_{j=1}^{k} F_{j}\left(x_{r}\right) \leqslant & n^{1-\sum_{j=1}^{k} \frac{1}{\lambda_{j}}} \prod_{j=1}^{\frac{k}{2}}\left\{( \sum _ { r = 1 } ^ { n } F _ { 2 j - 1 } ^ { \lambda _ { 2 j - 1 } } ( x _ { r } ) ) ^ { \frac { 1 } { \lambda _ { 2 j - 1 } } - \frac { 1 } { \lambda _ { 2 j } } } \left[\left(\left(\sum_{r=1}^{n} F_{2 j-1}^{\lambda_{2 j-1}}\left(x_{r}\right)\right)\left(\sum_{r=1}^{n} F_{2 j}^{\lambda_{2 j}}\left(x_{r}\right)\right)\right)^{2}\right.\right. \\
& \left.\left.-\left(\left(\sum_{r=1}^{n} F_{2 j-1}^{\lambda_{2 j-1}}\left(x_{r}\right) e_{r}\right)\left(\sum_{r=1}^{n} F_{2 j}^{\lambda_{2 j}}\left(x_{r}\right)\right)-\left(\sum_{r=1}^{n} F_{2 j-1}^{\lambda_{2 j-1}}\left(x_{r}\right)\right)\left(\sum_{r=1}^{n} F_{2 j}^{\lambda_{2 j}}\left(x_{r}\right) e_{r}\right)\right)^{2}\right]^{\frac{1}{2 \lambda_{2 j}}}\right\} .
\end{aligned}
$$

Hence, we have

$$
\begin{aligned}
& \sum_{r=1}^{n} \prod_{j=1}^{k} F_{j}\left(x_{r}\right) \frac{b-a}{n} \leqslant(b-a)^{1-\sum_{j=1}^{k} \frac{1}{\lambda_{j}}} \prod_{j=1}^{\frac{k}{2}}\left\{\left(\sum_{r=1}^{n} F_{2 j-1}^{\lambda_{2 j-1}}\left(x_{r}\right) \frac{b-a}{n}\right)^{\frac{1}{\lambda_{2 j-1}}-\frac{1}{\lambda_{2 j}}}\right. \\
& \times\left[\left(\left(\sum_{r=1}^{n} F_{2 j-1}^{\lambda_{2 j-1}}\left(x_{r}\right) \frac{b-a}{n}\right)\left(\sum_{r=1}^{n} F_{2 j}^{\lambda_{2 j}}\left(x_{r}\right) \frac{b-a}{n}\right)\right)^{2}\right. \\
& -\left(\left(\sum_{r=1}^{n} F_{2 j-1}^{\lambda_{2 j-1}}\left(x_{r}\right) e\left(x_{r}\right) \frac{b-a}{n}\right)\left(\sum_{r=1}^{n} F_{2 j}^{\lambda_{2 j}}\left(x_{r}\right) \frac{b-a}{n}\right)\right. \\
& \left.\left.\left.-\left(\sum_{r=1}^{n} F_{2 j-1}^{\lambda_{2 j-1}}\left(x_{r}\right) \frac{b-a}{n}\right)\left(\sum_{r=1}^{n} F_{2 j}^{\lambda_{2 j}}\left(x_{r}\right) e\left(x_{r}\right) \frac{b-a}{n}\right)\right)^{2}\right]^{\frac{1}{2 \lambda_{2 j}}}\right\} \text {. }
\end{aligned}
$$

In view of the hypotheses that $F_{j}(x), e(x)$ are positive Riemann integrable functions on $[a, b]$, we conclude that $F_{j}^{\lambda_{j}}(x), F_{j}^{\lambda_{j}}(x) e(x)$ are also integrable on $[a, b]$. Passing the limit as $n \rightarrow \infty$ in both sides of inequality (2.19), we obtain inequality (2.17).

Case (b). When $k$ is odd, by the same method as in the above Case (a), we have the inequality (2.18). The proof of Theorem 2.6 is completed.

From Theorem 2.6 we obtain the following generalizations and refinements of the generalized Hölder's inequality.

Corollary 2.7. Let $\mathrm{F}_{\mathrm{j}}(\mathrm{x}), \lambda_{j}, e(\mathrm{x})$ be as in Theorem 2.6, and let $\int_{\mathrm{a}}^{\mathrm{b}} \mathrm{F}_{j}^{\lambda_{j}}(\mathrm{x}) \mathrm{d} x \neq 0$. If $\mathrm{k}$ is even, then

$$
\begin{aligned}
\int_{a}^{b} \prod_{j=1}^{k} F_{j}(x) d x \leqslant & (b-a)^{1-\sum_{j=1}^{k} \frac{1}{\lambda_{j}}}\left[\prod_{j=1}^{k}\left(\int_{a}^{b} F_{j}^{\lambda_{j}}(x) d x\right)^{\frac{1}{\lambda_{j}}}\right] \\
& \times\left\{\prod_{j=1}^{\frac{k}{2}}\left[1-\frac{1}{2 \lambda_{2 j}}\left(\frac{\int_{a}^{b} F_{2 j-1}^{\lambda_{2 j-1}}(x) e(x) d x}{\int_{a}^{b} F_{2 j-1}^{\lambda_{2 j}-1}(x) d x}-\frac{\int_{a}^{b} F_{2 j}^{\lambda_{2 j}}(x) e(x) d x}{\int_{a}^{b} F_{2 j}^{\lambda_{2 j}}(x) d x}\right)^{2}\right]\right\} .
\end{aligned}
$$

If $\mathrm{k}$ is odd, then

$$
\int_{a}^{b} \prod_{j=1}^{k} F_{j}(x) d x \leqslant(b-a)^{1-\sum_{j=1}^{k} \frac{1}{\lambda_{j}}}\left[\prod_{j=1}^{k}\left(\int_{a}^{b} F_{j}^{\lambda_{j}}(x) d x\right)^{\frac{1}{\lambda_{j}}}\right]
$$




$$
\times\left\{\prod_{j=1}^{\frac{k-1}{2}}\left[1-\frac{1}{2 \lambda_{2 j}}\left(\frac{\int_{a}^{b} F_{2 j-1}^{\lambda_{2 j-1}}(x) e(x) d x}{\int_{a}^{b} F_{2 j-1}^{\lambda_{2 j-1}}(x) d x}-\frac{\int_{a}^{b} F_{2 j}^{\lambda_{2 j}}(x) e(x) d x}{\int_{a}^{b} F_{2 j}^{\lambda_{2 j}}(x) d x}\right)^{2}\right]\right\} .
$$

Now, we present the following generalizations of inequalities (1.4), (1.5), (1.8), (1.9) and (1.12).

Theorem 2.8. Let $A_{r j}>0,(r=1,2, \cdots, n, j=1,2, \cdots, m), \lambda_{1}>0, \lambda_{i}<0(i=2,3, \cdots, m)$, and $\sum_{j=1}^{m} \frac{1}{\lambda_{j}}>$ 0 , let $\tau=\max \left\{\sum_{j=1}^{m} \frac{1}{\lambda_{j}}, 1\right\}$, and let $1-e_{r}+e_{s} \geqslant 0(s=1,2, \cdots, n)$. Then

$$
\begin{aligned}
\sum_{r=1}^{n} \prod_{j=1}^{m} A_{r j} \geqslant & n^{1-\tau}\left(\sum_{r=1}^{n} A_{r 1}^{\lambda_{1}}\right)^{\frac{1}{\lambda_{1}}-\sum_{j=2}^{m} \frac{1}{\lambda_{j}}} \prod_{j=2}^{m}\left\{\left[\left(\sum_{r=1}^{n} A_{r 1}^{\lambda_{1}}\right)\left(\sum_{r=1}^{n} A_{r j}^{\lambda_{j}}\right)\right]^{2}\right. \\
& \left.-\left[\left(\sum_{r=1}^{n} A_{r 1}^{\lambda_{1}} e_{r}\right)\left(\sum_{r=1}^{n} A_{r j}^{\lambda_{j}}\right)-\left(\sum_{r=1}^{n} A_{r 1}^{\lambda_{1}}\right)\left(\sum_{r=1}^{n} A_{r j}^{\lambda_{j}} e_{r}\right)\right]^{2}\right\}^{\frac{1}{2 \lambda_{j}}} .
\end{aligned}
$$

Proof. We first consider the case (I) $\sum_{j=1}^{m} \frac{1}{\lambda_{j}} \geqslant 1$. Let $\sum_{j=1}^{m} \frac{1}{\lambda_{j}}=t(t \geqslant 1)$, which implies $\sum_{j=1}^{m} \frac{1}{t \lambda_{j}}=1$. Preforming some simple computations, we have

$$
\begin{aligned}
\sum_{r=1}^{n}( & \left.\prod_{j=1}^{k} A_{r j}\right) \sum_{s=1}^{n}\left(\prod_{i=1}^{k} A_{s i}\right)\left(1-e_{r}+e_{s}\right) \\
= & \sum_{r=1}^{n} \sum_{s=1}^{n}\left(\prod_{j=1}^{k} A_{r j}\right)\left(\prod_{i=1}^{k} A_{s i}\right)-\sum_{r=1}^{n} \sum_{s=1}^{n}\left(\prod_{j=1}^{k} A_{r j}\right)\left(\prod_{i=1}^{k} A_{s i}\right) e_{r} \\
& +\sum_{r=1}^{n} \sum_{s=1}^{n}\left(\prod_{j=1}^{k} A_{r j}\right)\left(\prod_{i=1}^{k} A_{s i}\right) e_{s} \\
= & \left(\sum_{r=1}^{n} \prod_{j=1}^{k} A_{r j}\right)^{2} .
\end{aligned}
$$

From inequality (2.3), we have

$$
\begin{aligned}
\sum_{s=1}^{n}( & \left.\prod_{i=1}^{m} A_{s i}\right) \sum_{r=1}^{n}\left(\prod_{j=1}^{m} A_{r j}\right)\left(1-e_{r}+e_{s}\right) \\
= & \sum_{s=1}^{n}\left(\prod_{i=1}^{m} A_{s i}\right) \sum_{r=1}^{n} \prod_{j=1}^{m} A_{r j}\left(1-e_{r}+e_{s}\right)^{\frac{1}{t} \lambda_{j}} \\
\geqslant & \sum_{s=1}^{n}\left(\prod_{i=1}^{m} A_{s i}\right)\left[\prod_{j=1}^{m}\left(\sum_{r=1}^{n} A_{r j}^{t} \lambda_{j}\left(1-e_{r}+e_{s}\right)\right)^{\frac{1}{\mathrm{t} \lambda_{j}}}\right] \\
= & \sum_{s=1}^{n}\left\{\left(A_{s 1}^{\mathrm{t} \lambda \lambda_{1}} \sum_{r=1}^{n} A_{r 1}^{\mathrm{t} \lambda_{1}}\left(1-e_{r}+e_{s}\right)\right)^{\frac{1}{\mathrm{t} \lambda_{1}}-\sum_{j=2}^{\mathrm{m}} \frac{1}{\mathrm{t} \lambda_{j}}}\left[\prod_{j=2}^{m}\left(A_{s 1}^{\mathrm{t} \lambda_{1}} \sum_{r=1}^{n} A_{r j}^{\mathrm{t} \lambda_{j}}\left(1-e_{r}+e_{s}\right)\right)^{\frac{1}{\mathrm{t} \lambda_{j}}}\right]\right. \\
& \left.\times\left[\prod_{j=2}^{m}\left(A_{s j}^{\mathrm{t} \lambda_{j}} \sum_{r=1}^{n} A_{r 1}^{\mathrm{t} \lambda_{1}}\left(1-e_{r}+e_{s}\right)\right)^{\frac{1}{\mathrm{t} \lambda_{j}}}\right]\right\} .
\end{aligned}
$$

Consequently, in view of $\left(\frac{1}{\mathrm{t} \lambda_{1}}-\sum_{j=2}^{m} \frac{1}{\mathrm{t} \lambda_{j}}\right)+\frac{1}{\mathrm{t} \lambda_{2}}+\frac{1}{\mathrm{t} \lambda_{3}}+\cdots+\frac{1}{\mathrm{t} \lambda_{\mathrm{m}}}+\frac{1}{\mathrm{t} \lambda_{2}}+\frac{1}{\mathrm{t} \lambda_{3}}+\cdots+\frac{1}{\mathrm{t} \lambda_{\mathrm{m}}}=1$, applying inequality (2.3) on the right side of (2.22), we find 


$$
\begin{aligned}
\sum_{s=1}^{n}( & \left.\prod_{i=1}^{m} A_{s i}\right) \sum_{r=1}^{n}\left(\prod_{j=1}^{m} A_{r j}\right)\left(1-e_{r}+e_{s}\right) \\
\geqslant & \left(\sum_{s=1}^{n} \sum_{r=1}^{n} A_{s 1}^{\mathrm{t} \lambda_{1}} A_{r 1}^{\mathrm{t} \lambda_{1}}\left(1-e_{r}+e_{s}\right)\right)^{\frac{1}{\mathrm{t} \lambda}-\sum_{j=2}^{\mathrm{m}} \frac{1}{\mathrm{t} \lambda_{j}}}\left[\prod_{j=2}^{\mathrm{m}}\left(\sum_{s=1}^{n} \sum_{r=1}^{n} A_{s 1}^{\mathrm{t} \lambda_{1}} A_{r j}^{\mathrm{t} \lambda_{j}}\left(1-e_{r}+e_{s}\right)\right)^{\frac{1}{\mathrm{t} \lambda_{j}}}\right] \\
& \times\left[\prod_{j=2}^{\mathrm{m}}\left(\sum_{s=1}^{n} \sum_{r=1}^{n} A_{s j}^{\mathrm{t} \lambda_{j}} A_{r 1}^{\mathrm{t} \lambda_{1}}\left(1-e_{r}+e_{s}\right)\right)^{\frac{1}{\mathrm{t} \lambda_{j}}}\right] .
\end{aligned}
$$

Moreover, using Lemma 2.2 together with $t \geqslant 1$, we find

$$
\begin{aligned}
& \left(\sum_{s=1}^{n} \sum_{r=1}^{n} A_{s 1}^{\mathrm{t} \lambda_{1}} A_{r 1}^{\mathrm{t} \lambda_{1}}\left(1-e_{r}+e_{s}\right)\right)^{\frac{1}{\mathrm{t} \lambda}-\sum_{j=2}^{m} \frac{1}{\mathrm{t} \lambda_{j}}}\left[\prod_{j=2}^{m}\left(\sum_{s=1}^{n} \sum_{r=1}^{n} A_{s 1}^{\mathrm{t} \lambda_{1}} A_{r j}^{\mathrm{t} \lambda_{j}}\left(1-e_{r}+e_{s}\right)\right)^{\frac{1}{\mathrm{t} \lambda_{j}}}\right] \\
& \times\left[\prod_{j=2}^{m}\left(\sum_{s=1}^{n} \sum_{r=1}^{n} A_{s j}^{t} \lambda_{j} A_{r 1}^{t \lambda_{1}}\left(1-e_{r}+e_{s}\right)\right)^{\frac{1}{\mathrm{t} \lambda} \lambda_{j}}\right] \\
& \geqslant\left(\sum_{s=1}^{n} \sum_{r=1}^{n}\left(1-e_{r}+e_{s}\right)\right)^{(1-t)\left(\frac{1}{t \lambda_{1}}-\sum_{j=2}^{m} \frac{1}{t \lambda_{j}}\right)}\left(\sum_{s=1}^{n} \sum_{r=1}^{n} A_{s 1}^{\lambda_{1}} A_{r 1}^{\lambda_{1}}\left(1-e_{r}+e_{s}\right)\right)^{\frac{1}{\lambda_{1}}-\sum_{j=2}^{m} \frac{1}{\lambda_{j}}} \\
& \times\left[\prod_{j=2}^{m}\left(\sum_{s=1}^{n} \sum_{r=1}^{n}\left(1-e_{r}+e_{s}\right)\right)^{\frac{1-t}{t_{j}}}\left(\sum_{s=1}^{n} \sum_{r=1}^{n} A_{s 1}^{\lambda_{1}} A_{r j}^{\lambda_{j}}\left(1-e_{r}+e_{s}\right)\right)^{\frac{1}{\lambda_{j}}}\right] \\
& \times\left[\prod_{j=2}^{m}\left(\sum_{s=1}^{n} \sum_{r=1}^{n}\left(1-e_{r}+e_{s}\right)\right)^{\frac{1-t}{t \lambda_{j}}}\left(\sum_{s=1}^{n} \sum_{r=1}^{n} A_{s j}^{\lambda_{j}} A_{r 1}^{\lambda_{1}}\left(1-e_{r}+e_{s}\right)\right)^{\frac{1}{\lambda_{j}}}\right] \\
& =\left(\sum_{s=1}^{n} \sum_{r=1}^{n}\left(1-e_{r}+e_{s}\right)\right)^{1-t}\left(\sum_{s=1}^{n} \sum_{r=1}^{n} A_{s 1}^{\lambda_{1}} A_{r 1}^{\lambda_{1}}\left(1-e_{r}+e_{s}\right)\right)^{\frac{1}{\lambda_{1}}-\sum_{j=2}^{m} \frac{1}{\lambda_{j}}} \\
& \times\left[\prod_{j=2}^{m}\left(\sum_{s=1}^{n} \sum_{r=1}^{n} A_{s 1}^{\lambda_{1}} A_{r j}^{\lambda_{j}}\left(1-e_{r}+e_{s}\right)\right)^{\frac{1}{\lambda_{j}}}\right]\left[\prod_{j=2}^{m}\left(\sum_{s=1}^{n} \sum_{r=1}^{n} A_{s j}^{\lambda_{j}} A_{r 1}^{\lambda_{1}}\left(1-e_{r}+e_{s}\right)\right)^{\frac{1}{\lambda_{j}}}\right] \\
& =n^{2-2 t}\left(\sum_{r=1}^{n} A_{r 1}^{\lambda_{1}}\right)^{\frac{2}{\lambda_{1}}-\sum_{j=2}^{m} \frac{2}{\lambda_{j}}}\left\{\prod _ { j = 2 } ^ { m } \left[\left(\sum_{s=1}^{n} \sum_{r=1}^{n} A_{s 1}^{\lambda_{1}} A_{r j}^{\lambda_{j}}\left(1-e_{r}+e_{s}\right)\right)\right.\right. \\
& \left.\left.\times\left(\sum_{s=1}^{n} \sum_{r=1}^{n} A_{s j}^{\lambda_{j}} A_{r 1}^{\lambda_{1}}\left(1-e_{r}+e_{s}\right)\right)\right]^{\frac{1}{\lambda_{j}}}\right\} \\
& =n^{2-2 t}\left(\sum_{r=1}^{n} A_{r 1}^{\lambda_{1}}\right)^{\frac{2}{\lambda_{1}}-\sum_{j=2}^{m} \frac{2}{\lambda_{j}}} \\
& \times\left\{\prod _ { j = 2 } ^ { m } \left[\left(\sum_{s=1}^{n} A_{s 1}^{\lambda_{1}} \sum_{r=1}^{n} A_{r j}^{\lambda_{j}}-\sum_{s=1}^{n} A_{s 1}^{\lambda_{1}} \sum_{r=1}^{n} A_{r j}^{\lambda_{j}} e_{r}+\sum_{s=1}^{n} A_{s 1}^{\lambda_{1}} e_{s} \sum_{r=1}^{n} A_{r j}^{\lambda_{j}}\right)\right.\right. \\
& \left.\left.\times\left(\sum_{s=1}^{n} A_{s j}^{\lambda_{j}} \sum_{r=1}^{n} A_{r 1}^{\lambda_{1}}-\sum_{s=1}^{n} A_{s j}^{\lambda_{j}} \sum_{r=1}^{n} A_{r 1}^{\lambda_{1}} e_{r}+\sum_{s=1}^{n} A_{s j}^{\lambda_{j}} e_{s} \sum_{r=1}^{n} A_{r 1}^{\lambda_{1}}\right)\right]^{\frac{1}{\lambda_{j}}}\right\} \\
& =n^{2-2 t}\left(\sum_{r=1}^{n} A_{r 1}^{\lambda_{1}}\right)^{\frac{2}{\lambda_{1}}-\sum_{j=2}^{m} \frac{2}{\lambda_{j}}}\left\{\prod _ { j = 2 } ^ { m } \left[\left(\left(\sum_{r=1}^{n} A_{r 1}^{\lambda_{1}}\right)\left(\sum_{r=1}^{n} A_{r j}^{\lambda_{j}}\right)\right)^{2}\right.\right. \\
& \left.\left.-\left(\left(\sum_{r=1}^{n} A_{r 1}^{\lambda_{1}}\right)\left(\sum_{r=1}^{n} A_{r j}^{\lambda_{j}} e_{r}\right)-\left(\sum_{r=1}^{n} A_{r 1}^{\lambda_{1}} e_{r}\right)\left(\sum_{r=1}^{n} A_{r j}^{\lambda_{j}}\right)\right)^{2}\right]^{\frac{1}{\lambda_{j}}}\right\} \text {. }
\end{aligned}
$$


Combining inequalities (2.21), (2.23) and (2.24) leads to inequality (2.20) immediately.

Nextly, we consider the case (II) $0<\sum_{j=1}^{m} \frac{1}{\lambda_{j}} \leqslant 1$. On the one hand, by the inequality (2.1), we have

$$
\begin{aligned}
\sum_{s=1}^{n}( & \left.\prod_{i=1}^{m} A_{s i}\right) \sum_{r=1}^{n}\left(\prod_{j=1}^{m} A_{r j}\right)\left(1-e_{r}+e_{s}\right)^{\sum_{j=1}^{m} \frac{1}{\lambda_{j}}} \\
= & \sum_{s=1}^{n} \sum_{r=1}^{n}\left(\prod_{i=1}^{m} A_{s i}\right)\left(\prod_{j=1}^{m} A_{r j}\right)\left(1-e_{r}+e_{s}\right)^{\sum_{j=1}^{m} \frac{1}{\lambda_{j}}} \\
\leqslant & {\left[\sum_{s=1}^{n} \sum_{r=1}^{n}\left(\prod_{i=1}^{m} A_{s i}\right)\left(\prod_{j=1}^{m} A_{r j}\right)\right]^{1-\sum_{j=1}^{m} \frac{1}{\lambda_{j}}} } \\
& \times\left[\sum_{s=1}^{n} \sum_{r=1}^{n}\left(\prod_{i=1}^{m} A_{s i}\right)\left(\prod_{j=1}^{m} A_{r j}\right)\left(1-e_{r}+e_{s}\right)\right]^{\sum_{j=1}^{m} \frac{1}{\lambda_{j}}} \\
= & {\left[\sum_{s=1}^{n} \sum_{r=1}^{n}\left(\prod_{i=1}^{m} A_{s i}\right)\left(\prod_{j=1}^{m} A_{r j}\right)\right]^{1-\sum_{j=1}^{m} \frac{1}{\lambda_{j}}} } \\
& \times\left[\sum_{s=1}^{n} \sum_{r=1}^{n}\left(\prod_{i=1}^{m} A_{s i}\right)\left(\prod_{j=1}^{m} A_{r j}\right)-\sum_{s=1}^{n} \sum_{r=1}^{n}\left(\prod_{i=1}^{m} A_{s i}\right)\left(\prod_{j=1}^{m} A_{r j}\right) e_{r}\right. \\
& \left.+\sum_{s=1}^{n} \sum_{r=1}^{n}\left(\prod_{i=1}^{m} A_{s i}\right)\left(\prod_{j=1}^{m} A_{r j}\right) e_{s}\right]^{\sum_{j=1}^{m} \frac{1}{\lambda_{j}}} \\
= & {\left[\sum_{s=1}^{n} \sum_{r=1}^{n}\left(\prod_{i=1}^{m} A_{s i}\right)\left(\prod_{j=1}^{m} A_{r j}\right)\right]^{1-\sum_{j=1}^{m} \frac{1}{\lambda_{j}}}\left[\sum_{s=1}^{n} \sum_{r=1}^{n}\left(\prod_{i=1}^{m} A_{s i}\right)\left(\prod_{j=1}^{m} A_{r j}\right)\right]^{\sum_{j=1}^{m} \frac{1}{\lambda_{j}}} } \\
= & \sum_{s=1}^{n} \sum_{r=1}^{n}\left(\prod_{i=1}^{m} A_{s i}\right)\left(\prod_{j=1}^{m} A_{r j}\right) \\
= & {\left[\sum_{r=1}^{n}\left(\prod_{j=1}^{m} A_{r j}\right)\right]^{2} \cdot }
\end{aligned}
$$

On the other hand, by the inequality (2.3), we obtain

$$
\begin{aligned}
\sum_{s=1}^{n}( & \left.\prod_{i=1}^{m} A_{s i}\right) \sum_{r=1}^{n}\left(\prod_{j=1}^{m} A_{r j}\right)\left(1-e_{r}+e_{s}\right)^{\sum_{j=1}^{m} \frac{1}{\lambda_{j}}} \\
= & \sum_{s=1}^{n}\left(\prod_{i=1}^{m} A_{s i}\right) \sum_{r=1}^{n} \prod_{j=1}^{m} A_{r j}\left(1-e_{r}+e_{s}\right)^{\frac{1}{\lambda_{j}}} \\
\geqslant & \sum_{s=1}^{n}\left(\prod_{i=1}^{m} A_{s i}\right)\left[\prod_{j=1}^{m}\left(\sum_{r=1}^{n} A_{r j}^{\lambda_{j}}\left(1-e_{r}+e_{s}\right)\right)^{\frac{1}{\lambda_{j}}}\right] \\
= & \sum_{s=1}^{n}\left\{\left(A_{s 1}^{\lambda_{1}} \sum_{r=1}^{n} A_{r 1}^{\lambda_{1}}\left(1-e_{r}+e_{s}\right)\right)^{\frac{1}{\lambda_{1}}-\sum_{j=2}^{m} \frac{1}{\lambda_{j}}}\left[\prod_{j=2}^{m}\left(A_{s 1}^{\lambda_{1}} \sum_{r=1}^{n} A_{r j}^{\lambda_{j}}\left(1-e_{r}+e_{s}\right)\right)^{\frac{1}{\lambda_{j}}}\right]\right. \\
& \left.\times\left[\prod_{j=2}^{m}\left(A_{s j}^{\lambda_{j}} \sum_{r=1}^{n} A_{r 1}^{\lambda_{1}}\left(1-e_{r}+e_{s}\right)\right)^{\frac{1}{\lambda_{j}}}\right]\right\} .
\end{aligned}
$$


Consequently, according to $\left(\frac{1}{\lambda_{1}}-\sum_{j=2}^{m} \frac{1}{\lambda_{j}}\right)+\frac{1}{\lambda_{2}}+\frac{1}{\lambda_{3}}+\cdots+\frac{1}{\lambda_{m}}+\frac{1}{\lambda_{2}}+\frac{1}{\lambda_{3}}+\cdots+\frac{1}{\lambda_{m}} \leqslant 1$, by using the inequality (2.3) on the right side of (2.26), we observe that

$$
\begin{aligned}
\sum_{s=1}^{n}( & \left.\prod_{i=1}^{m} A_{s i}\right) \sum_{r=1}^{n}\left(\prod_{j=1}^{m} A_{r j}\right)\left(1-e_{r}+e_{s}\right)^{\sum_{j=1}^{m} \frac{1}{\lambda_{j}}} \\
\geqslant & \left(\sum_{s=1}^{n} \sum_{r=1}^{n} A_{s 1}^{\lambda_{1}} A_{r 1}^{\lambda_{1}}\left(1-e_{r}+e_{s}\right)\right)^{\frac{1}{\lambda_{1}}-\sum_{j=2}^{m} \frac{1}{\lambda_{j}}}\left[\prod_{j=2}^{m}\left(\sum_{s=1}^{n} \sum_{r=1}^{n} A_{s 1}^{\lambda_{1}} A_{r j}^{\lambda_{j}}\left(1-e_{r}+e_{s}\right)\right)^{\frac{1}{\lambda_{j}}}\right] \\
& \times\left[\prod_{j=2}^{m}\left(\sum_{s=1}^{n} \sum_{r=1}^{n} A_{s j}^{\lambda_{j}} A_{r 1}^{\lambda_{1}}\left(1-e_{r}+e_{s}\right)\right)^{\frac{1}{\lambda_{j}}}\right] \\
= & \left(\sum_{r=1}^{n} A_{r 1}^{\lambda_{1}}\right)^{\frac{2}{\lambda_{1}}-\sum_{j=2}^{m} \frac{2}{\lambda_{j}}}\left\{\prod _ { j = 2 } ^ { m } \left[\left(\sum_{s=1}^{n} \sum_{r=1}^{n} A_{s 1}^{\lambda_{1}} A_{r j}^{\lambda_{j}}\left(1-e_{r}+e_{s}\right)\right)\right.\right. \\
& \left.\times\left(\sum_{s=1}^{n} \sum_{r=1}^{n} A_{s j}^{\lambda_{j}} A_{r 1}^{\lambda_{1}}\left(1-e_{r}+e_{s}\right)\right)^{\frac{1}{\lambda_{j}}}\right\} \\
= & \left(\sum_{r=1}^{n} A_{r 1}^{\lambda_{1}}\right)^{\frac{2}{\lambda_{1}}-\sum_{j=2}^{m} \frac{2}{\lambda_{j}}}\left\{\prod _ { j = 2 } ^ { m } \left[\left(\sum_{s=1}^{n} A_{s 1}^{\lambda_{1}} \sum_{r=1}^{n} A_{r j}^{\lambda_{j}}-\sum_{s=1}^{n} A_{s 1}^{\lambda_{1}} \sum_{r=1}^{n} A_{r j}^{\lambda_{j}} e_{r}+\sum_{s=1}^{n} A_{s 1}^{\lambda_{1}} e_{s} \sum_{r=1}^{n} A_{r j}^{\lambda_{j}}\right)\right.\right. \\
& \left.\left.\times\left(\sum_{s=1}^{n} A_{s j}^{\lambda_{j}} \sum_{r=1}^{n} A_{r 1}^{\lambda_{1}}-\sum_{s=1}^{n} A_{s j}^{\lambda_{j}} \sum_{r=1}^{n} A_{r 1}^{\lambda_{1}} e_{r}+\sum_{s=1}^{n} A_{s j}^{\lambda_{j}} e_{s} \sum_{r=1}^{n} A_{r 1}^{\lambda_{1}}\right)\right]^{\frac{1}{\lambda_{j}}}\right\} \\
= & \left(\sum_{r=1}^{n} A_{r 1}^{\lambda_{1}}\right)^{\frac{2}{\lambda_{1}}-\sum_{j=2}^{m} \frac{2}{\lambda_{j}}}\left\{\prod _ { j = 2 } ^ { m } \left[\left(\left(\sum_{r=1}^{n} A_{r 1}^{\lambda_{1}}\right)\left(\sum_{r=1}^{n} A_{r j}^{\lambda_{j}}\right)\right)^{2}\right.\right. \\
& \left.\left.-\left(\left(\sum_{r=1}^{n} A_{r 1}^{\lambda_{1}}\right)\left(\sum_{r=1}^{n} A_{r j}^{\lambda_{j}} e_{r}\right)-\left(\sum_{r=1}^{n} A_{r 1}^{\lambda_{1}} e_{r}\right)\left(\sum_{r=1}^{n} A_{r j}^{\lambda_{j}}\right)\right)^{2}\right]^{\frac{1}{\lambda_{j}}}\right\} .
\end{aligned}
$$

Combining inequalities (2.25) and (2.27) leads to inequality (2.20) immediately. The proof of Theorem 2.8 is completed.

From Theorem 2.8 and Lemma 2.1, we obtain the following generalizations and refinements of generalized Hölder's inequality (2.3).

Corollary 2.9. Let $A_{r j}, \lambda_{j}, e_{r}$ be as in Theorem 2.8 , let $\tau=\max \left\{\sum_{j=1}^{m} \frac{1}{\lambda_{j}}, 1\right\}$, and let $\sum_{r=1}^{n} A_{r j}^{\lambda_{j}} \neq 0$. Then

$$
\sum_{r=1}^{n} \prod_{j=1}^{m} A_{r j} \geqslant n^{1-\tau}\left[\prod_{j=1}^{m}\left(\sum_{r=1}^{n} A_{r j}^{\lambda_{j}}\right)^{\frac{1}{\lambda_{j}}}\right]\left\{\prod_{j=2}^{m}\left[1-\frac{1}{2 \lambda_{j}}\left(\frac{\sum_{r=1}^{n} A_{r 1}^{\lambda_{1}} e_{r}}{\sum_{r=1}^{n} A_{r 1}^{\lambda_{1}}}-\frac{\sum_{r=1}^{n} A_{r j}^{\lambda_{j}} e_{r}}{\sum_{r=1}^{n} A_{r j}^{\lambda_{j}}}\right)^{2}\right]\right\} .
$$

Theorem 2.10. Let $\mathrm{F}_{j}(\mathrm{x}), e(\mathrm{x})$ be integrable functions defined on $[\mathrm{a}, \mathrm{b}]$ and $\mathrm{F}_{\mathrm{j}}(\mathrm{x})>0,1-e(\mathrm{x})+e(\mathrm{y}) \geqslant 0$ for all $x, y \in[a, b]$, and let $\sum_{j=1}^{m} \frac{1}{\lambda_{j}} \geqslant 1$. If $\lambda_{1}>0, \lambda_{j}<0(j=2,3, \cdots, m)$, then

$$
\begin{aligned}
\int_{a}^{b} \prod_{j=1}^{m} F_{j}(x) d x \geqslant & (b-a)^{1-\sum_{j=1}^{m} \frac{1}{\lambda_{j}}}\left(\int_{a}^{b} F_{1}^{\lambda_{1}}(x) d x\right)^{\frac{1}{\lambda_{1}}-\sum_{j=2}^{m} \frac{1}{\lambda_{j}}} \prod_{j=2}^{m}\left[\left(\int_{a}^{b} F_{1}^{\lambda_{1}}(x) d x \int_{a}^{b} F_{j}^{\lambda_{j}}(x) d x\right)^{2}\right. \\
& -\left(\int_{a}^{b} F_{1}^{\lambda_{1}}(x) e(x) d x \int_{a}^{b} F_{j}^{\lambda_{j}}(x) d x-\int_{a}^{b} F_{1}^{\lambda_{1}}(x) d x \int_{a}^{b} F_{j}^{\lambda_{j}}(x) e(x) d x\right)^{\frac{1}{2 \lambda_{j}}} .
\end{aligned}
$$


Proof. Making similar method as in the proof of Theorem 2.6 by using inequality (2.20), we have the desired inequality (2.28).

Corollary 2.11. Let $\mathrm{F}_{j}(x), \lambda_{j}, e(x)$ be as in Theorem 2.10, and let $\int_{a}^{b} F_{j}^{\lambda_{j}}(x) \mathrm{d} x \neq 0$. Then, we have the following generalization and refinement of generalized Hölder's inequality (1.9).

$$
\begin{aligned}
\int_{a}^{b} \prod_{j=1}^{m} F_{j}(x) d x \geqslant & (b-a)^{1-\sum_{j=1}^{m} \frac{1}{\lambda_{j}}}\left[\prod_{j=1}^{m}\left(\int_{a}^{b} F_{j}^{\lambda_{j}}(x) d x\right)^{\frac{1}{\lambda_{j}}}\right] \\
& \times\left\{\prod_{j=2}^{m}\left[1-\frac{1}{2 \lambda_{j}}\left(\frac{\int_{a}^{b} F_{1}^{\lambda_{1}}(x) e(x) d x}{\int_{a}^{b} F_{1}^{\lambda_{1}}(x) d x}-\frac{\int_{a}^{b} F_{j}^{\lambda_{j}}(x) e(x) d x}{\int_{a}^{b} F_{j}^{\lambda_{j}}(x) d x}\right)^{2}\right]\right\}
\end{aligned}
$$

Proof. Making similar arguments as in the proof of Corollary 2.5, we have the desired inequality (2.29).

\section{Acknowledgment}

The authors would like to express their sincere thanks to the anonymous referees for their great efforts to improve this paper.

This work was supported by the Application Basic Research Plan Key Basic Research Project of Hebei Province of China (No. 16964213D), the Fundamental Research Funds for the Central Universities (No. 2015ZD29, 13ZD19) and the Higher School Science Research Funds of Hebei Province of China (No. Z2015137).

\section{References}

[1] H. Agahi, Y. Ouyang, R. Mesiar, E. Pap, M. Štrboja, Hölder and Minkowski type inequalities for pseudo-integral, Appl. Math. Comput., 217 (2011), 8630-8639. 1

[2] E. F. Beckenbach, R. Bellman, Inequalities, Second revised printing, Ergebnisse der Mathematik und ihrer Grenzgebiete, Neue Folge, Band 30 Springer-Verlag, New York, Inc., (1965). 2.1

[3] G. H. Hardy, J. E. Littlewood, G. Pólya, Inequalities, Reprint of the 1952 edition, Cambridge Mathematical Library, Cambridge University Press, Cambridge, (1988). 2.2

[4] K. Hu, On an inequality and its applications, Sci. Sinica, 24 (1981), 1047-1055. 1, 1

[5] J.-C. Kuang, Applied inequalities, Shandong Science and Technology Press, Jinan, China, (2004). 1

[6] J. Matkowski, A converse of the Hölder inequality theorem, Math. Inequal. Appl., 12 (2009), 21-32. 1

[7] J.-F. Tian, Extension of Hu Ke's inequality and its applications, J. Inequal. Appl., 2011 (2011), 14 pages. 1, 1

[8] J.-F. Tian, Reversed version of a generalized sharp Hölder's inequality and its applications, Inform. Sci., 201 (2012), 61-69. 1

[9] J.-F. Tian, Property of a Hölder-type inequality and its application, Math. Inequal. Appl., 16 (2013), 831-841.

[10] J.-F. Tian, New property of a generalized Hölder's inequality and its applications, Inform. Sci., 288 (2014), 45-54.

[11] J.-F. Tian, Properties of generalized Hölder's inequalities, J. Math. Inequal., 9 (2015), 473-480.

[12] J.-F. Tian, M.-H. Ha, Properties of generalized sharp Hölder's inequalities, J. Math. Inequal., 11 (2017), 511-525.

[13] J.-F. Tian, X.-M. Hu, A new reversed version of a generalized sharp Hölder's inequality and its applications, Abstr. Appl. Anal., 2013 (2013), 9 pages. 1

[14] J.-F. Tian, W. Pedrycz, New refinements of generalized Hölder's inequality and their applications, Math. Inequal. Appl., 19 (2016), 805-822. 1

[15] P. M. Vasić, J. E. Pečarić, On the Jensen inequality for monotone functions, An. Univ. Timişoara Ser. Ştiinţ. Mat., 17 (1979), 95-104. 2.3

[16] S.-H. Wu, Generalization of a sharp Hölder's inequality and its application, J. Math. Anal. Appl., 332 (2007), 741-750. 1, 1

[17] C.-J. Zhao, W.-S. Cheung, On reverse Minkowski-type inequalities, Mediterr. J. Math., 12 (2015), 1085-1094.

[18] C.-J. Zhao, W. S. Cheung, Hölder's reverse inequality and its applications, Publ. Inst. Math. (Beograd) (N.S.), 99 (2016), 211-216. 1 